\title{
Materiales audiovisuales para el estudio de cuestiones de Derecho de Familia y Sucesiones a partir de la jurisprudencia de Estrasburgo $^{1}$
}

\author{
Catalina Pons-Estel Tugores. Profesora Contratada Doctora interina de Derecho Civil \\ Universidad de las Islas Baleares \\ E-mail: catalina.pons-estel@uib.es
}

Marcos González Sánchez. Profesor Titular de Derecho Eclesiástico del Estado

Universidad Autónoma de Madrid

E-mail: marcos.gonzalez@uam.es

\begin{abstract}
This paper presents the teaching innovation project in which we have opted for the promotion of active learning methodologies. The reverse pedagogy has been used and the students have been the ones who have assumed the elaboration of audiovisual material that will support them and their future colleagues (we want these tools to be available and can be used during several academic courses) to address the study of current issues of Family Law and Inheritance and Donations. In particular, the jurisprudence of Strasbourg has been studied since it is the main current instrument for the legal protection of human rights at the international level.
\end{abstract}

Keywords: audiovisual, jurisprudence, methodology, learning, coordination, family, successions.

\begin{abstract}
Resumen
En el proyecto de innovación docente que presentamos se ha optado por el impulso de las metodologías activas de aprendizaje. Se ha usado la pedagogía inversa y los alumnos han sido quienes han asumido la elaboración de material audiovisual que les servirá de apoyo a ellos y a sus compañeros futuros (pues tenemos intención de que estas herramientas tengan perdurabilidad y las podamos usar a lo largo de varios cursos académicos) para abordar el estudio de cuestiones actuales del Derecho de
\end{abstract}

\footnotetext{
${ }^{1}$ Comunicación realizada en el marco del Proyecto de Innovación Docente "Materiales audiovisuales para el estudio de cuestiones de Derecho de Familia y Sucesiones a partir de la jurisprudencia de Estrasburgo" (PID 171817) perteneciente a los proyectos de innovación y mejora de la calidad docente de la Universidad de las Islas Baleares (2017-2018), siendo la investigadora principal la Dra. Catalina Pons-Estel Tugores y formando parte del Proyecto el Dr. Marcos González Sánchez, el Dr. Pedro A. Munar Bernat, el Dr. Salvador Pérez Álvarez, la Dra. Beatriz Verdera y la Dra. Francisca $\mathrm{M}^{\mathrm{a}}$ Rosselló Rubert.
} 
Familia y de Sucesiones y Donaciones. Especialmente, se ha estudiado la jurisprudencia de Estrasburgo al ser el principal instrumento actual para la tutela jurídica de los derechos humanos en el plano internacional.

Palabras clave: audiovisual, jurisprudencia, metodología, aprendizaje, coordinación, familia, sucesiones.

\section{Introducción}

La configuración actual de los planes de estudios exige una innovación continua en la metodología docente que se aplica en nuestras aulas. No solo los profesores, sino también los alumnos y la sociedad en general, demandamos unas mejoras y evolución constantes en la manera de "enseñar-aprender". Quienes integramos el proyecto de Innovación Docente que ahora presentamos somos profesores de Facultades de Derecho y nuestra propuesta se basa en los siguientes elementos: a) El estudio de la jurisprudencia de Estrasburgo en tres cuestiones concretas del Derecho de Familia y Sucesiones: derecho a contraer matrimonio, adopción y custodia. Hay que tener en cuenta que el TEDH es probablemente el principal instrumento actual para la tutela jurídica de los derechos humanos en el plano internacional y su influencia va mucho más allá del Consejo de Europa y su repercusión es cada vez mayor en la modificación del Derecho interno de los Estados sometidos a su jurisdicción. Las sentencias del TEDH relativas al derecho al respeto a la vida privada y familiar (artículo 8 del CEDH) son cada vez más abundantes; b) El impulso de las metodologías activas de aprendizaje, ya que en una parte de nuestra propuesta hemos usado la pedagogía inversa y han sido nuestros alumnos quienes han asumido la elaboración del material audiovisual; c) el uso de herramientas TIC; y, d) la interdisciplinariedad de la materia, pues colaboraremos en el proyecto profesores de tres áreas de conocimiento, trabajando en equipo y coordinando nuestra labor docente.

\section{Contexto en el que se ha desarrollado el proyecto}

El equipo que ha desarrollado la experiencia docente que detallamos está integrado por profesores de Derecho Civil, Derecho Eclesiástico del Estado y Derecho Mercantil de tres Universidades distintas (Universidad de las Islas Baleares, Universidad Autónoma de Madrid y Universidad Nacional de Educación a Distancia), que imparten diversas asignaturas de sus disciplinas. Se ha trabajado coordinadamente logrando una comparativa de los diversos grupos y del aprendizaje conseguido por los alumnos.

En la Universidad de las Islas Baleares el proyecto se ha desarrollado en un grupo de Persona y Familia ( $2^{\circ}$ de Grado en Derecho) con 84 alumnos matriculados impartido por la Dra. Catalina Pons-Estel Tugores, profesora de Derecho Civil; un grupo de Derecho Eclesiástico del Estado (asignatura optativa de $4^{\circ}$ curso) con 50 alumnos matriculados, también impartido por la Dra. Pons-Estel; dos grupos de Donaciones y Sucesiones ( $4^{\circ} \mathrm{de}$ Grado en Derecho) con 60 y 45 alumnos matriculados, impartido por el Dr. Pedro A. Munar Bernat, Catedrático de Derecho Civil; un grupo de Persona y Familia (2 $2^{\circ}$ de Grado 
en Administración y Dirección de Empresas y Derecho) con 45 alumnos matriculados impartido por la Dra. Beatriz Verdera, profesora de Derecho Civil y un grupo de Nociones básicas de Derecho ( $1^{\circ}$ de Grado en Economía) con 10 alumnos matriculados impartido por la Dra. Francisca $\mathrm{M}^{\mathrm{a}}$ Rosselló Rubert, Ayudante de Derecho Mercantil. En la Universidad Autónoma de Madrid se ha trabajado con 5 alumnos de Trabajo Fin de Grado tutorizados por el Dr. Marcos González Sánchez, Profesor Titular de Derecho Eclesiástico del Estado. Por su parte, en la Universidad Nacional de Educación a Distancia se ha trabajado con el Dr. Salvador Pérez Álvarez, Profesor Titular de Derecho Eclesiástico del Estado y organizador de un Seminario celebrado en su Universidad con la finalidad de compartir y transferir los resultados logrados con el Proyecto.

Los conocimientos en materia de Persona y Familia, Donaciones y Sucesiones y Jurisprudencia del Tribunal Europeo de Derechos Humanos son muy distintos en un grupo o en otro, debido -fundamentalmente- al curso concreto que están estudiando los alumnos. Precisamente por eso, el estudio de estas materias se ha realizado de forma progresiva, según los conocimientos jurídicos del alumnado. Ello ha supuesto que los resultados alcanzados han sido diversos dependiendo del curso académico; pero, en todos ellos se ha pretendido fomentar el razonamiento lógico y el espíritu crítico.

Persona y Familia es una asignatura que, por un lado introduce en el estudio del Derecho de la Persona (personalidad jurídica, capacidad natural, capacidad de obrar, modificaciones judiciales de la capacidad de obrar, representación, etc.); y, por otro, aborda el Derecho de Familia (instituciones jurídicas que regulan las relaciones personales y patrimoniales entre los miembros de la familia y en sus relaciones con terceros). Precisamente el concepto de Familia ha sufrido una auténtica revolución en los últimos tiempos y en ella nos encontramos con familias "tradicionales", monoparentales, uniones heterosexuales u homosexuales (inscritas y no inscritas), familias reconstituidas, familias ocasionales, etc. Además, también a día de hoy hay instituciones que están viviendo una etapa crítica a la espera de una nueva regulación, como es el caso de la filiación y la dificultad que conlleva en ocasiones cohonestar la realidad biológica y la realidad jurídica de la misma.

El Derecho Eclesiástico del Estado es una rama especializada del ordenamiento jurídico que se ocupa de cómo el Derecho del Estado regula la exteriorización del fenómeno religioso en la sociedad (tanto en su dimensión individual como en la colectiva). También se pretende la familiarización del alumnado con las fuentes, los principios informadores, la legislación específica y la jurisprudencia propia del Derecho Eclesiástico, con el objetivo de poder profundizar en aquellas cuestiones que resulten de interés, especialmente interrelacionando los conocimientos de esta materia con el resto de las del ordenamiento jurídico.

Para ello, los contenidos temáticos que se trabajan son: a) concepto, evolución histórica y fuentes del Derecho Eclesiástico; b) concepto, contenido, límites, titularidad y protección jurídica del derecho fundamental de libertad religiosa; c) la dimensión individual del derecho de libertad religiosa (enseñanza, asistencia religiosa, objeción de conciencia, matrimonio canónico y acatólico -junto con su eficacia civil-); y, d) la dimensión colectiva del derecho de libertad religiosa (personalidad jurídica de las entidades religiosas, 
patrimonio cultural de las confesiones religiosas, financiación y régimen fiscal de las confesiones religiosas, etc.).

La asignatura Donaciones y Sucesiones se imparte en cuarto curso del grado en Derecho y en ella se analizan instituciones de gran arraigo social como son las derivadas del Derecho sucesorio, que llevan a concretar el destino y consecuencias del patrimonio de una persona después de su fallecimiento. En dicha asignatura se estudian distintas instituciones como pueden ser el título sucesorio, el concepto mismo de herencia y el estudio de todo el proceso que lleva a la adquisición de la misma. Y, respecto al Derecho de Donaciones se trata de concretar el procedimiento a seguir para determinar la transmisión voluntaria de bienes sin que medie un precio. Por tanto, se estudian las donaciones remuneratorias, modales, las mortis causa, con cláusula de reversión, las liberalidades de uso, etc.

Por su parte, Nociones básicas de Derecho es una asignatura de formación básica cuya función es ofrecer una panorámica de la regulación jurídica de la vida cotidiana. Sirve para tener una visión previa y general de todo aquello que, a lo largo de la carrera, se estudiará con mayor profundidad. Fundamentalmente pretende que se conozcan y comprendan -en un nivel básico- las principales nociones, instituciones y principios jurídicos.

Los Trabajos Fin de Grado son una materia globalizadora que pretende la evaluación integrada de las competencias específicas y transversales del Grado en Derecho. El objetivo es conseguir que los alumnos sean aptos para realizar un trabajo de forma autónoma e individual aplicando e integrando las competencias adquiridas durante la carrera.

\section{Objetivos}

El objetivo principal es conseguir que los alumnos adquieran conciencia del papel que juega la jurisprudencia del TEDH en el ámbito privado familiar y sucesorio. A su vez, queremos que se familiaricen con los criterios seguidos hasta la fecha por el citado Tribunal y que valoren el impacto de sus resoluciones. En otro orden de cosas, sabemos por experiencia propia, que la mejor forma de estudiar cualquier temática es teniéndola que explicar. Es por eso que estamos convencidos de que al pedir a nuestros estudiantes que preparen una cuestión muy concreta para elaborar el material audiovisual que servirá de apoyo a sus compañeros actuales y futuros (pues tenemos intención de que estas herramientas tengan perdurabilidad y las podamos usar a lo largo de varios cursos académicos), en realidad, les estamos empujando a conocer a fondo los temas que van a trabajar. Además, al recibir las explicaciones de sus propios compañeros, los estudiantes muestran una mejor predisposición a escuchar y a aprender. Incluso, más curiosidad y participación activa.

El objetivo secundario es elaborar un material de apoyo audiovisual, que quedará a disposición del alumnado, a través del Aula digital. Creemos que mediante las distintas actividades que hemos planteado durante el curso 2017-2018, hemos logrado trabajar las distintas competencias propias del Grado en Derecho. Así, queremos contribuir a la formación integral de los alumnos como juristas y a posibilitarles que en un futuro gocen de total autonomía, de forma que sean capaces de enfrentarse a nuevos problemas y de 
encontrar la forma de solucionarlos. Para ello, es básico que puedan conseguir información jurídica (ya sea derecho positivo, doctrina, jurisprudencia, etc.), a través de las fuentes instrumentales, incluidas las electrónicas. De este modo, será necesario que, a nivel de usuario, manejen las herramientas electrónicas básicas. Con nuestra propuesta, les ayudaremos -mediante la ejemplificación- a comprender, analizar y sistematizar la jurisprudencia de Estrasburgo en la materia concreta del Derecho de familia, así como a conocer de qué forma se aplica. Y no solo para resolver conflictos actuales, sino también para prever las potenciales consecuencias jurídicas de la ejecución de un determinado acto. La idea de que sean los propios alumnos quienes elaboren los recursos audiovisuales nace de la convicción de los miembros de este Proyecto de la importancia capital de la comunicación. Así, hay que saber exponer ideas, propuestas, soluciones jurídicas y hacerlo de forma ordenada, precisa, concisa -ya sea oralmente, ya sea por escrito- adaptando nuestro discurso a las circunstancias. Pensamos que la preparación y exposición por parte de los alumnos, de cuestiones muy concretas, no solo apoyará estas competencias, sino que, además, potenciará la actitud crítica, el ser capaz de contrastar cualquier información jurídica, analizando las consecuencias políticas, económicas y sociales. Por cuanto venimos indicando, este proyecto promueve especialmente la metodología activa de aprendizaje; la coordinación entre diversas materias de un mismo Grado; el uso de herramientas TIC en la metodología de enseñanza-aprendizaje; la evaluación de la calidad docente y de los procesos de evaluación y aprendizaje de los alumnos; la orientación tutorial; la realización de actividades de formación complementaria; e incluso el uso de lenguas extranjeras, por cuanto las resoluciones del TEDH aparecen en inglés y en francés y -lógicamente- la promoción de la elaboración de material didáctico.

\section{Desarrollo de la innovación}

\subsection{Metodología}

Nuestro planteamiento metodológico consta de varias fases, que, cronológicamente han seguido el siguiente desarrollo: A) De cada cuestión que hemos trabajado con los alumnos hemos elaborado una carpeta con material (normativo, doctrinal, jurisprudencial, pericial, etc.) que está disponible online, en la página de nuestras asignaturas en el Aula digital, a fin de que puedan trabajarlo con anterioridad y posterioridad a nuestras explicaciones en clase; B) Hemos abordado los temas mediante el sistema tradicional de clase magistral; C) Hemos propuesto unas palabras clave e ideas básicas de cada tema; D) Hemos formulado preguntas relacionadas con la temática trabajada para que activaran el espíritu crítico de los alumnos; E) Hemos realizado prácticas; F) Hemos planteado foros de debate; G) Hemos pedido a los alumnos que, individualmente, elaboraran un vídeo del tema asignado, de una duración comprendida entre 1.5-3.5 minutos; $\mathrm{H}$ ) Hemos subido este material audiovisual a la plataforma, a fin de que todos puedan disponer de él; I) Realizaremos un Mesa redonda abierta al público en general, pues pensamos que así será más enriquecedora- sobre las cuestiones más candentes que hemos trabajado y lo haremos mediante exposiciones breves (15 minutos aprox.) y sugerentes, a fin de potenciar el debate ulterior entre todos los asistentes y los ponentes, pues los alumnos estarán muy formados acerca de las cuestiones 
que se expondrán y estamos convencidos de que disfrutarán de participar. A lo largo de todo el íterin, los alumnos han contado con unas tutorías específicas para hacer un seguimiento de su proceso de aprendizaje, así como para orientarles en todo aquello que hayan necesitado.

\subsection{Plan de trabajo}

El plan de trabajo en el que han participado todos los profesores implicados en el proyecto ha sido el siguiente:

a) Selección, bajo la dirección de su tutor, de 25 sentencias del TEDH sobre derecho a contraer matrimonio, adopción, custodia y ajuste a derecho de las sentencias de nulidad matrimonial canónica y posterior elaboración de una breve síntesis de cada una de ellas por parte de los alumnos de TFG de la Universidad Autónoma de Madrid. Posteriormente, este material se transfirió a los alumnos de la Universidad de las Islas Baleares de los grupos implicados en el proyecto que nos ocupa.

b) Explicación por parte de los profesores al alumnado de la Universidad balear de la trascendencia e influencia de la jurisprudencia del Tribunal de Estrasburgo en la modificación del Derecho interno de los Estados sometidos a su jurisdicción, haciendo especial hincapié en la materia específica de cada docente (persona y familia, derecho de libertad religiosa, etc.).

c) Elaboración por parte del profesorado de un dossier con material sobre la jurisprudencia del Tribunal de Estrasburgo (artículos doctrinales, sentencias, etc.).

d) Elaboración de la propuesta de los trabajos y actividades a realizar por los alumnos.

e) Puesta a disposición del alumnado de la Universidad de las Islas Baleares del material proporcionado por los alumnos de TFG de la Universidad Autónoma de Madrid, así como del dossier con material y la propuesta de trabajos y actividades a realizar.

f) Estudio por parte de los alumnos del material recibido y selección de un tema.

g) Elaboración por parte de los estudiantes de un borrador de trabajo.

h) Realización de tutorías individualizadas para evaluar la viabilidad de las propuestas de los alumnos y para guiar la realización de los trabajos (tanto las fichas como el material audiovisual) hasta su culminación.

i) Celebración de una sesión tutorizada por el personal de Campus Extens a los alumnos de los grupos de Persona y Familia y de Derecho Eclesiástico del Estado para resolver las dudas técnicas que hubieran podido surgir en la elaboración del material audiovisual.

j) Acceso a todo el alumnado implicado en el proyecto a las fichas y a los materiales audiovisuales realizados.

k) Valoración en clase de las actividades desarrolladas. 


\section{Resultados}

En el marco de este proyecto de innovación se ha realizado una Mesa redonda en el Aula de Grados de la Facultad de Derecho de la Universidad de las Islas Baleares -moderada por el Dr. Munar Bernat, miembro del equipo docente del proyecto- en la que han intervenido los Dres. González Sánchez y Pons-Estel Tugores -también miembros de dicho proyecto de innovación- y se ha tratado la cuestión de la Jurisprudencia del TEDH en el reconocimiento de efectos civiles a los matrimonies religiosos. Además, se ha celebrado un Seminario en la UNED con la finalidad de compartir y transferir los resultados logrados con el Proyecto.

Por otro lado, en el ámbito de la docencia, las actividades realizadas con el alumnado han sido:

a) Explicaciones teóricas en dos sesiones de clase.

b) Incorporación de una carpeta específica de la temática (legislación, doctrina, jurisprudencia, prensa, etc.) en la página de la asignatura en el Aula digital, poniendo a su disposición una carpeta con abundante material formativo, que hemos ido ampliando y actualizando a medida que avanzaba el curso.

c) Prácticas en una clase.

d) Asistencia a la mesa redonda en la que participaron como ponentes dos miembros del equipo docente del proyecto.

e) Foro a través de la página de la asignatura en el Aula digital -abierto durante 21 días-, en el que los alumnos han tenido que reflexionar y responder individualmente sobre cuál es el papel que juega la jurisprudencia del TEDH en relación a la jurisprudencia española relativa al Derecho de Familia y Sucesiones. Precisamente las intervenciones en este Foro han generado un intenso debate entre los participantes y tenemos que subrayar que la participación del alumnado ha sido masiva y la interactuación entre los distintos miembros del foro, continua.

f) Y como colofón, se ha logrado el objetivo de la elaboración de materiales audiovisuales. Concretamente, esta actividad constó de dos partes:

A) Una primera, en la que cada alumno escogió el tema y la sentencia del TEDH que deseó (siempre que fuera de la materia de Derecho de Familia o de Donaciones y Sucesiones) y completó una ficha al respecto en la que debían constar:

-Los datos de la sentencia: Tribunal, fecha de la sentencia y sujetos (recurrente y recurrido).

-El tema principal. Objeto del recurso.

-El tema o temas secundarios.

-El resumen de los antecedentes fácticos.

-El resumen de la fundamentación jurídica.

-Las disposiciones legales aplicadas.

-El fallo de la sentencia.

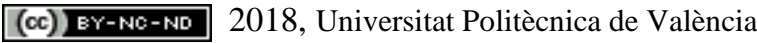

Congreso In-Red (2018) 
-La valoración personal.

-La ubicación del material audiovisual elaborado por el alumno, a fin de que todos los participantes del grupo pudieran acceder al mismo.

-La relación de fuentes de información manejadas.

La extensión de la ficha no debía superar las 3 páginas. Lógicamente, con anterioridad el alumno habrá tenido que subir la grabación a la red, pues en la ficha ya tenía que indicarse la ubicación del material audiovisual elaborado por el alumnado.

B) Una segunda en la que cada alumno llevó a cabo una grabación (utilizando su teléfono móvil o su tablet) en la que abordó la explicación del tema que había escogido, así como el planteamiento jurisprudencial del TEDH acerca del mismo. La duración de la grabación abarcó desde un minuto y medio (duración mínima) hasta tres minutos y medio (duración máxima).

Como resultado final, hemos conseguido 54 grabaciones que suponen un material valiosísimo no solo para los alumnos que han participado en este proyecto, sino también para las futuras promociones que podrán beneficiarse de este gran trabajo. Además, la aceptación por los propios alumnos participantes en este proyecto ha sido muy satisfactoria, por cuanto el 82\% de ellos así lo han manifestado en una encuesta realizada.

\section{Conclusiones}

Tras la finalización de las distintas actividades llevadas a cabo en el marco del proyecto de innovación que ahora presentamos, los profesores integrantes del mismo nos reunimos para hacer una valoración del mismo y, muy brevemente, podemos reseñar las siguientes conclusiones:

a) Esta iniciativa ha resultado muy enriquecedora para los alumnos, fundamentalmente por dos motivos: porque ha supuesto una toma de contacto con compañeros de otra Universidad y porque ha posibilitado una relación de trabajo y complemento entre alumnos de distintos cursos.

b) La elaboración del material audiovisual ha sido muy motivadora porque ha supuesto que en el parte final del trabajo han podido utilizar instrumentos tan propios de su día a día y tan populares como el teléfono móvil o la tableta.

c) El hecho de que los alumnos hayan tenido que elaborar material didáctico útil para sus compañeros les ha obligado a tener que estudiar y comprender en profundidad una temática que, de entrada, es muy compleja. En cambio, se han metido tanto en su papel de “docentes” que ni siquiera se han percatado de la cantidad de horas que han invertido en la consecución del objetivo final que era el material audiovisual.

d) La multidisciplinariedad del proyecto por la participación en el mismo de profesores de tres disciplinas distintas ha proporcionado una riqueza supletoria durante todo el proceso de trabajo y, sin duda, también en el resultado final. El intercambio de opiniones entre 
profesionales que se centran en aspectos distintos, hace que tengamos una perspectiva más amplia.

e) De entrada, trescientos alumnos han participado en la elaboración de este material audiovisual y, por tanto, se benefician del mismo de forma directa. Pero, este trabajo va más allá, pues estos recursos audiovisuales serán utilizados durante los próximos cursos como material de apoyo. Por esto, resulta difícil cuantificar el número total de alumnos que de forma más o menos inmediata podrán usar estos recursos. Lo que sí tenemos claro es que serán muchos.

f) Para los profesores implicados en el proyecto esta iniciativa ha supuesto muchísimo trabajo extra. No obstante, también nosotros nos hemos sentido muy motivados al ver a los alumnos tan implicados en el mismo y con tantas ansias de hacer las cosas bien.

\section{Referencias}

AA.VV. (1993). Marriage and religion in Europe. Milano: Giuffrè Editore.

AlCARAZ Ramos, M. (2000). "Familia y matrimonio en las constituciones de los Estados de la Unión Europea” en AA.VV. Estudios en homenaje al Profesor Martínez Valls. Alicante: Universidad de Alicante, vol. II, pp. 749-760.

CAÑAMARES ARRIBAS, S. (2014). "El derecho al matrimonio en la jurisprudencia del TEDH” en Derecho y Religión. vol. 9, pp. 245-260.

COOPER, S.L. (2010). "Marriage, Family, Discrimination \& Contradiction: An Evaluation of the Legacy and Future of the European Court of Human Rights' Jurisprudence on LGBT Rights” en German Law Journal. vol. 12, pp. 1747-1761.

FARNÓs AMORÓs, E. (2015). "La reproducción asistida ante el Tribunal de Estrasburgo margen de apreciación v. necesidad de armonización” en AA.VV. Treinta años de reproducción asistida en España Recurso electrónico una mirada interdisciplinaria a un fenómeno global y actual. Madrid: Ministerio de Justicia, pp. 175-202.

GonZÁLEZ SÁNCHEZ, M. (2008). La incidencia de los acuerdos internacionales sobre derechos fundamentales en la jurisprudencia de Derecho Eclesiástico del Tribunal Constitucional. Navarra: Civitas.

NANClARES VALLE, J. (2015). "Convivencia entre personas del mismo sexo e interés público en la jurisprudencia del Tribunal Europeo de Derechos Humanos” en Persona y Derecho, vol. 72, 2015, pp. 109-132.

NOVALES AlquÉZAR, M.A. (2015). "La filiación de matrimonio homosexual en España en el contexto europeo”, en AA.VV. Nuevas orientaciones del derecho civil en Europa. Navarra: Dykinson.

Paniza Fullana, A. (2017), Realidad biológica versus realidad jurídica: el necesario replanteamiento de la filiación. Navarra: Aranzadi. 
Polo Sabau, J.R. (2006). Matrimonio y Constitución ante la Reforma del Derecho de Familia. Navarra: Civitas.

SANZ CABALLERO, S. (2004/2005). "Unmarried cohabiting couples before the European court of Human Rights; parity with marriage?” en Columbia Journal of European Law. pp. 151 y ss.

(cc) EY-NC-ND 2018, Universitat Politècnica de València 